\title{
Introduction of an ENT emergency-safe boot camp into postgraduate surgical training in the Republic of Ireland
}

\author{
Oludare Alabi ${ }^{1,3} \cdot$ Rhodri Hill $^{1,2} \cdot$ Michael Walsh $^{1,4} \cdot$ Camilla Carroll $^{1,3}$
}

Received: 15 December 2020 / Accepted: 26 February 2021 / Published online: 8 March 2021

(c) Royal Academy of Medicine in Ireland 2021

\begin{abstract}
Background Otolaryngology (ENT) is a craft surgical specialty, which presents significant challenges for the novice surgical trainee (NST), when managing patients with potentially life-threatening conditions. We assess current evidence behind intensive, simulationrich training programmes or "boot camps". We outline an ENT emergency-safe boot camp for NSTs and the impact this can have on trainees' competence when dealing with ENT emergencies.

Methods We designed a standardized "ENT emergency-safe boot camp", aimed at NSTs with a view to up-skilling them in the management of 3 critical clinical scenarios namely post-thyroidectomy haematoma, post-tonsillectomy haemorrhage and epistaxis. All core surgical trainees (CSTs) $(n=54)$ on the National Surgical Training programme at the Royal College of Surgeons (RCSI) were enrolled on the boot camp. A pre- and post-boot camp anonymised self-assessment was completed by all participants.

Results Trainees' self-assessment demonstrated an increase in median competence scores from $3 / 5$ to $4 / 5$ for management of post-thyroidectomy haematoma and epistaxis. There was a greater increase in self-assessed competence scores from $2 / 5$ to $4 / 5$ for the management of post-tonsillectomy haemorrhage. Ninety-two percent of respondents said the course added to their previous knowledge, $70 \%$ of respondents said it improved their technical skills and $85 \%$ of participants said the course gave them more confidence with handling ENT related on-call problems.

Conclusion Study participants expressed an increase in confidence when dealing with specific ENT emergencies, following boot camp training. This correlates with published data. Patient safety should be enhanced, as a result of participation in this dedicated ENT emergency-safe boot camp.
\end{abstract}

Keywords Boot camp $\cdot$ Medical education $\cdot$ Otolaryngology $\cdot$ Simulation training $\cdot$ Surgical training

\section{Introduction}

The commencement of a new surgical job or training post can be a challenging experience for junior doctors. Prior inexperience can lead to a reduction in the standard of patient care at the start of the new academic year [1]. This

Oludare Alabi

oludarealabi@rcsi.ie

1 Royal College of Surgeons in Ireland, 123 St Stephen's Green, Dublin, Ireland

2 Ireland University Hospital Waterford, Waterford, Ireland

3 Royal Victoria Eye and Ear Hospital, Adelaide Road, Dublin, Ireland

4 Health Service Executive, Dublin, Ireland is especially evident in craft surgical specialties like otolaryngology (ENT), which medical graduates commonly feel underprepared for [2]. The need to reduce patient errors, combined with the difficulty of providing all elements of training while complying with working time restrictions, has led to an increase in the use of surgical boot camps [3]. Boot camp typically involves focused skills training in a range of simulated clinical scenarios. It requires the novice trainee to act in real time and to receive instant feedback which they can use to practice and improve.

Several studies have shown the benefit of a boot camp to surgical trainees by increasing confidence in handling emergencies, providing technical skills through simulation and identifying when early escalation is required [3-5]. Skills acquisition can also be accelerated in a boot camp setting with reported retention rates of up to 6 months [6-8]. 
As expected, the long-term benefit of boot camp training has been shown to be short-lived without subsequent deliberate practice and further distributive learning [9]. Deliberate practice, which involves engaging in structured practice that focuses on improvement as opposed to repetition, has been shown to produce superior results amongst trainees than prior standard models of training. This is likely as a result of goal directed practice in a structured environment and realtime feedback $[10,11]$. Ultimately, a boot camp model with subsequent provision of opportunities to access structured simulated training will provide the most long-term benefit. Despite this, the benefit of accelerated learning that a boot camp provides during the initial period of relative inexperience still remains [6].

At our institution, core surgical trainees (CSTs) have commenced their training with a simulation-based surgical boot camp; however, this was lacking an ENT component. Currently, ENT emergencies' training is provided to trainees after commencement of the training programme. Trainees will benefit more from undergoing such training prior to commencement of such posts [8].

We aimed to create an "ENT emergency-safe boot camp", which would equip trainees with necessary skills to competently manage commonly encountered ENT emergencies. The overall goal of this was to provide a safe transition to ENT practice for trainees by equipping them with skills needed to manage common ENT emergencies. We also aimed to increase their levels of confidence in managing such cases through simulation-based practice.

\section{Methods}

On consultation with surgical faculty and the national surgical training program, challenging areas for novice ENT trainees and potentially life-threatening emergencies that are commonly encountered were identified. A standardised intensive, simulation-rich training programme or "boot camp" aimed at novice surgical trainees was designed with a view to up-skilling them in the management of 3 critical clinical scenarios namely:

1. Evacuation of a post-thyroidectomy haematoma.

2. Nasal packing in the presence of severe epistaxis.

3. Management of post-tonsillectomy haemorrhage.

These are situations to which a CST may be commonly called to provide initial management.

A competence-based educational model was used, based on adult learning theories, with a focus on skills transfer in a simulated learning environment. The "boot camp" was delivered over $4 \mathrm{~h}$. It included a blended learning approach with interactive didactic teaching as well as practical skills stations with simulation and virtual reality airway models. All 54 CSTs on the National Surgical Training programme at the Royal College of Surgeons in Ireland (RCSI) were enrolled on the boot camp and training was delivered over four iterations. Prior to the boot camp, each trainee received literature on the management of the aforementioned clinical scenarios.

The boot camp faculty consisted of 3 legitimate Senior ENT experts, 2 surgical trainees and simulation centre staff. The programme was delivered in the National Surgical Skills Centre, at the Royal College of Surgeons in Ireland. The clinical management of each scenario was based on a Cochrane literature review of best practice and was proto$\mathrm{col}$ and procedural based. An anonymised questionnaire was completed by each candidate upon completion of the boot camp. It assessed their prior surgical experience, their previous encounters managing the above scenarios and selfassessed impact of the boot camp training.

\section{Results}

Fifty-six percent of respondents had 2 years of surgical experience with $44 \%$ having 3-5 years of surgical experience. Despite this, most of the respondents had few clinical encounters with scenarios being covered. Seventyfive percent of respondents had encountered epistaxis less than 5 times; this was $98 \%$ for post-tonsillar haemorrhage and $90 \%$ for post-thyroidectomy haematomas.

Trainees' self-assessment based on the reflective questionnaire showed an increase in median confidence scores from $3 / 5$ to $4 / 5$ for epistaxis and post-thyroidectomy bleeds. There was a greater increase in self-assessed competence scores from $2 / 5$ to $4 / 5$ for the management of post-tonsillectomy bleeds. Trainees reported interactive lectures to be the most beneficial part of the boot camp with $48 \%$ preferring them compared with $29 \%$ for simulated case scenarios and $23 \%$ for simulated procedural skills training. Ninety-two percent (46/50) of respondents said the course added to their previous knowledge while $6 \%$ were neutral. Seventy percent $(38 / 54)$ of respondents agreed that it improved their technical skills; $22 \%$ were neutral regarding this. Eighty-five percent (45/53) of respondents felt they would be more confident managing ENT related issues when on call, $11 \%$ were neutral.

\section{Discussions}

Evidence has shown that boot camp training is an effective way to accelerate clinical competence in the novice surgical trainee. Being a surgical trainee on-call, it is important 
to be able to provide initial management of ENT emergencies. We successfully introduced an "ENT emergency-safe boot camp" for NSTs into the Postgraduate Surgical Training Programme, in the Republic of Ireland. Our experience supported previous findings on the positive impact of boot camp on increasing participants' confidence in handling situations they had limited exposure to with the biggest increase in confidence levels seen in the management of post-tonsillectomy bleeds. In addition to confidence levels, the majority of trainees reported increased overall knowledge and technical skills. The participants who were ambivalent about the impact of the boot camp on their confidence and skills had more surgical experience and were more likely to have the ability to manage the aforementioned scenarios prior to the boot camp.

While it is unlikely that the skills and confidence gained from the boot camp will last without clinical exposure to these scenarios or deliberate practice, the benefits at the start of the training year still remain. Making simulation equipment available for constant practice and refreshing of skills will aid retainment; however, this is limited by resources available.

It is also notable that participants reported interactive lectures to be the most effective part of the boot camp in aiding learning. This was perhaps due to inexperience in ENT and a larger knowledge gap than practical skills gap in the management of these emergencies. Overall, participants expressed satisfaction with the boot camp. It is expected that skills acquisition will translate into better patient outcomes, when NSTs encounter these scenarios in the clinical setting.

Finally, as medical training faces challenges all around the world in light of the COVID-19 public health situation, there is a need to maintain training while allowing adherence to public health guidelines of social distancing. This is increasing the demand for simulation-based training. While the boot camp was started before the COVID-19 pandemic, it is even more relevant now. This simulation-based boot camp will help maintain emergency ENT training within the constraints of the current pandemic.

Acknowledgements We acknowledge the support of the RCSI National Surgical Training Programme, as well as the assistance of the staff at the RCSI Centre for Simulation Education for their efforts towards the successful implementation of the Boot camp.
Data availability Results of the qualitative questionnaire are available with human or animal subjects. Facilities and equipment were provided by the Royal College of Surgeons in Ireland.

\section{Declarations}

Ethical approval Was not required for this study. It was a qualitative survey and did not involve experimenting with human or animal subjects.

Conflict of interest The authors declare that they have no conflicts of interest.

\section{References}

1. Inaba K, Recinos G, Teixeira PG et al (2010) Complications and death at the start of the new academic year: is there a July phenomenon? J Trauma 68(1):19-22

2. Ferguson GR, Bacila IA, Swamy M (2016) Does current provision of undergraduate education prepare UK medical students in ENT? A systematic literature review. BMJ Open 6(4):e010054

3. Blackmore C, Austin J, Lopushinsky SR, Donnon T (2014) Effects of postgraduate medical education "boot camps" on clinical skills, knowledge, and confidence: a meta-analysis. J Grad Med Educ 6(4):643-652

4. Esterl RM Jr, Henzi DL, Cohn SM (2006) Senior medical student "Boot Camp": can result in increased self-confidence before starting surgery internships. CurrSurg 63(4):264-268

5. Malekzadeh S, Malloy KM, Chu EE et al (2011) ORL emergencies boot camp: using simulation to onboard residents. Laryngoscope 121(10):2114-2121

6. Parent RJ, Plerhoples TA, Long EE et al (2010) Early, intermediate, and late effects of a surgical skills "boot camp" on an objective structured assessment of technical skills: a randomized controlled study. J Am CollSurg 210(6):984-989

7. Laeeq K, Pandian V, Skinner M et al (2010) Learning curve for competency in flexible laryngoscopy. Laryngoscope 120(10):1950-1953

8. Okusanya OT, Kornfield ZN, Reinke CE et al (2012) The effect and durability of a pregraduation boot cAMP on the confidence of senior medical student entering surgical residencies. J SurgEduc 69(4):536-543

9. Moulton CA, Dubrowski A, Macrae H (2006) Teaching surgical skills: what kind of practice makes perfect?: a randomized, controlled trial. Ann Surg 244(3):400-409

10. Bhatti NI, Ahmed A (2015) Improving skills development in residency using a deliberate-practice and learner-centered model. Laryngoscope 125(Suppl 8):S1-14

11. Ericsson KA (2004) Deliberate practice and the acquisition and maintenance of expert performance in medicine and related domains. Acad Med 79(10 Suppl):S70-81 